\title{
Quantification of resistant alleles in the $\beta$-tubulin gene of field strains of gastrointestinal nematodes and their relation with the faecal egg count reduction test
}

\author{
Myriam Esteban-Ballesteros ${ }^{1,2}$, Francisco A. Rojo-Vázquez 1,2, Philip J. Skuce ${ }^{3}$, Lynsey Melville ${ }^{3}$, \\ Camino González-Lanza ${ }^{2}$ and María Martínez-Valladares ${ }^{1,2^{*}}$
}

\begin{abstract}
Background: Benzimidazole (BZ) resistance in gastrointestinal nematodes is associated with a single nucleotide polymorphism (SNP) at codons 167, 198 and 200 in the isotype 1 of beta-tubulin gene although in some species these SNPs have also been associated with resistance to macrocyclic lactones. In the present study we compared the levels of resistance in Teladorsagia circumcincta and Trichostrongylus colubriformis by means of the faecal egg reduction test (FECRT) and the percentage of resistant alleles obtained after pyrosequencing. The study was conducted in 10 naturally infected sheep flocks. Each flock was divided into three groups: i) group treated with albendazole (ABZ); ii) group treated with ivermectin (IVM); iii) untreated group. The number of eggs excreted per gram of faeces was estimated at day 0 and 14 post-treatment.

Results: Resistance to ABZ was observed in 12.5\% (1/8) of the flocks and to IVM in 44.4\% (4/9) of them. One flock was resistant to both drugs according to FECRT. Coprocultures were performed at the same dates to collect L3 for DNA extraction from pooled larvae and to determine the resistant allele frequencies by pyrosequencing analysis. In T. circumcincta, SNPs were not found at any of the three codons before treatment; after the administration of ABZ, SNPs were present only in two different flocks, one of them with a frequency of $23.8 \%$ at SNP 167, and the other $13.2 \% \%$ at SNP 198. In relation to T. colubriformis, we found the SNP200 before treatment in 33.3\% (3/9) of the flocks with values between 48.5 and $87.8 \%$. After treatment with ABZ and IVM, the prevalence of this SNP increased to 75 and $100 \%$ of the flocks, with a mean frequency of $95.1 \%$ and $82.6 \%$, respectively.
\end{abstract}

Conclusion: The frequencies observed for SNP200 in T. colubriformis indicate that the presence of resistance is more common than revealed by the FECRT.

Keywords: Sheep, Teladorsagia circumcincta, Trichostrongylus colubriformis, Anthelmintic resistance, FECRT, Single nucleotide polymorphism, Beta-tubulin, Pyrosequencing

\footnotetext{
*Correspondence: mmarva@unileon.es

'Departamento de Sanidad Animal, Facultad de Veterinaria, Universidad de

León, Campus de Vegazana s/n, 24071 León, Spain

${ }^{2}$ Instituto de Ganadería de Montaña (CSIC-Universidad de León), Finca

Marzanas, Grulleros, 24346 León, Spain

Full list of author information is available at the end of the article
} 


\section{Background}

Infections by gastrointestinal nematodes (GIN) are a serious problem for extensive systems of sheep farming worldwide. GIN can cause serious losses in animal production since they affect the production of milk, wool and meat, and also interfere with reproduction [1]. The most prevalent GIN species infecting sheep in temperate areas of the world are Teladorsagia circumcincta, Trichostrongylus spp., Haemonchus contortus, Chabertia ovina and Cooperia spp.

The usual mode of controlling GIN infections in ruminants is by chemotherapy and the most commonly used anthelmintics are grouped into 3 families: benzimidazoles (BZs), imidazothiazoles and macrocyclic lactones (MLs). Recently, two new chemical groups have been introduced to the market, namely, the aminoacetonitrile derivative (monepantel) [2] and the spiroindole (derquantel), in combination with abamectin [3]. Anthelmintics have been used with great success in the past. However, their frequent use and the underdosing of animals has favored the emergence of anthelmintic resistance (AR), among other factors. There are some reports describing AR against all anthelmintic groups worldwide, even against the most recent anthelmintic drug, monepantel, in New Zealand, The Netherlands and Uruguay in $T$. circumcincta, Trichostrongylus colubriformis and $H$. contortus [4-7]. Therefore, the high prevalence of AR to several drugs against GIN in small ruminants continues to threaten the viability of small ruminant farms [8].

The BZs and MLs are the major groups of anthelmintics used to control GIN infections in some countries like Spain [9]. The mode of action of BZ involves binding to $\beta$ tubulin and disrupting microtubule polymerization of tubulin [10-13]. Genetic studies have shown that BZ resistance is associated with a single nucleotide polymorphism (SNP) in the gene encoding isotype-1 $\beta$ tubulin. The substitution of a phenylalanine (Phe, TTC) for a tyrosine (Tyr, TAC) at codon 200 (F200Y) has been linked to BZ resistance in $H$. contortus, $T$. colubriformis, $T$. circumcincta, C. oncophora and O. ostertagi [14]. Less frequently, the same SNP was found at codon 167 (F167Y) in resistant strains of $H$. contortus, $T$ circumcincta and $O$. ostertagi [15]. Furthermore, a point mutation of alanine (Ala, GCA) to glutamine (Glu, GAA) at codon 198 has been described in resistant strains of $H$. contortus, $C$. oncophora and O. ostertagi [16].

Previous assays have shown that resistant strains of $H$. contortus also carried the resistant allele at codons 200 and 167 after treatment with an ML, specifically, ivermectin (IVM). These results suggest a possible association between resistance mechanisms in ML and BZ [17].

Since AR is increasing recently around the world $[8,18,19]$, its control is required with the aim to use anthelmintic drugs more effectively and sustainably and to avoid the development of new resistant strains. The faecal egg count reduction test (FECRT) is the most widely used diagnostic method for the detection of AR in vivo [20, 21]. However, this technique lacks sensitivity and is not able to detect resistance when the level of genetically resistant individuals in the population is below $\sim 25 \%$ [22]. Therefore, the development of new in vitro techniques is necessary for an early diagnosis of AR.

In this context, the aim of the present study was the measurement of the frequency of the resistant allele at codons 200, 198 and 167 of isotype-1 $\beta$-tubulin in GIN field isolates of sheep flocks from the Northwest of Spain before and after administration of a BZ and/or ML anthelmintic.

\section{Methods}

\section{Faecal egg count reduction test (FECRT)}

The study was conducted on 10 sheep flocks located in the province of León, Northwest Spain. In each flock, two groups of 10 sheep, naturally infected by GIN, were selected. Each group was treated with a $\mathrm{BZ}$, albendazole (ABZ) $(7.5 \mathrm{mg} / \mathrm{kg} \quad \mathrm{bw})$, or IV $(0.2 \mathrm{mg} / \mathrm{kg} \mathrm{bw})$. Faecal samples were collected on day 0 and day 14 post-treatment (pt). The number of eggs per gram of faeces (EPG) was determined by the modified McMaster method [23]. The faecal egg count reduction was calculated according to the recommendations of WAAVP (World Association for the Advancement of Veterinary Parasitology) [24] and using the following formula:

$$
\begin{aligned}
\operatorname{FECRT} \%= & (\text { Arithmetic mean epg day } 0 \\
& - \text { Arithmetic mean epg days } \\
& +10-14) / \text { Arithmetic mean epg day } 0 \\
& \times 100
\end{aligned}
$$

When the percentage reduction in egg count was $<90 \%$, the flock was considered resistant to the anthelmintic; if the faecal reduction was between 90 and 95\%, the flock was classified as borderline or suspicious of resistance, and when values were higher than 95\%, the flock was considered susceptible to the anthelmintic.

From each group, pooled faeces were cultured on days 0 and 14 pt to recover third stage larvae (L3). After collecting the cultures, a minimum of 100 L3 per culture were identified using the morphological keys in MAFF [23]; in flocks with a reduction of $100 \%$ we recovered a few L3, between 20 and 225. The remainder was stored at $-20{ }^{\circ} \mathrm{C}$ until the DNA extraction was carried out. 


\section{DNA extraction}

DNA was extracted from each L3 pool, before and after treatment, using the Speed Tools Tissue DNA Extraction kit (Biotools), according to the manufacturer's instructions. The DNA samples were stored at $-20{ }^{\circ} \mathrm{C}$ until used.

\section{Determination of allele frequencies}

Two pairs of PCR primers were designed to amplify two fragments of the gene encoding the $\beta$-tubulin of $T$. circumcincta including the SNPs 167 and 198/200, respectively (Table 1). For Trichostrongylus colubriformis, we only designed one pair of primers to amplify a fragment encompassing codons 198 and 200 jointly (Table 1).

With the aim to amplify these three fragments, we firstly carried out a PCR with the primers described in Table 1, but without biotinylation. Cycling conditions were $95{ }^{\circ} \mathrm{C}$ for $10 \mathrm{~min}$ followed by 40 cycles of $95{ }^{\circ} \mathrm{C}$ for $30 \mathrm{~s}$, Tm for $30 \mathrm{~s}$ and $72{ }^{\circ} \mathrm{C}$ for $45 \mathrm{~s}$ followed by $10 \mathrm{~min}$ at $72{ }^{\circ} \mathrm{C}$ and $4{ }^{\circ} \mathrm{C}$ to finish using Taq DNA polymerase MasterMix 2x, $\mathrm{MgCl}_{2}$ 2,0 mM (Biotools, Madrid, Spain). PCR products were run on a $1.5 \%$ agarose gel, and the corresponding band was cut out and purified using PCR Clean-Up SpeedTools kit (Biotools, Madrid, Spain). The final elution volume was $50 \mu \mathrm{l}$. Then, this same PCR was carried out again in a $50 \mu \mathrm{l}$ reaction volume but, in this case, one of the primers of each pair was labeled with biotin at the $5^{\prime}$, as shown in Table 1 . Prior to pyrosequencing, a $7 \mu$ l aliquot of each PCR product was tested by agarose gel electrophoresis.

The specificity of the primers used to amplify these regions was confirmed initially after the amplification of the two regions in $T$. circumcincta adult worms and of one region in T. colubriformis adult worms. These PCRs were run in the same way as previously described but using $1 \mu \mathrm{l}$ of DNA template. The resulting bands were excised from an agarose gel 1,5\% and purified using PCR Clean-Up SpeedTools kit (Biotools) and then were sequenced in the "Laboratorio de Técnicas Instrumentales" (University of León, Spain).

Pyrosequencing of the three resulting PCR fragments, targeting SNPs 167 and 198/200 in T. circumcincta and SNPs $198 / 200$ in T. colubriformis, was carried out using the sequencing primers (Seq) (Table 1). The sequencing primer for the SNP167 of T. circumcincta was previously described by Skuce et al. [25]. The pyrosequencing assay was carried out using a PyroMark ID Pyrosequencer (Biotage, Sweden) according to the manufacturer's recommendations. After the initial PCR amplification, $40 \mu \mathrm{l}$ of PCR product was added to $37 \mu \mathrm{l} 2 \mathrm{x}$ Binding buffer (Biotage, Sweden), $3 \mu$ l streptavidin sepharose beads (Roche) in a 96 well plate and then agitated for $5 \mathrm{~min}$ at room temperature to allow binding of biotin-labelled DNA to the beads. The beads were processed using the sample preparation tool and reagents (Biotage, Sweden) dispensed into the assay plate with $40 \mu \mathrm{l}$ of $0.4 \mu \mathrm{M}$ sequencing primer per well. Positive controls representing gDNA extracted from susceptible and resistant individual adults from each species were included in each assay.

The determination of the allele frequencies was carried out at least 3 times for each sample and the arithmetic mean was calculated. The frequencies of the resistant allele with values equal to or lower than $<5 \%$ were considered as technical background and, therefore, not classed as resistant.

\section{Results}

Faecal egg count reduction test (FECRT)

The results of the FECRT carried out in the 10 sheep flocks are shown in Tables 3 and 4.

Table 1 Primer sequences for T. circumcincta SNPs 167 and 198/200, and T. colubriformis SNP 198/200, and sequence primers for both species

\begin{tabular}{|c|c|c|c|c|}
\hline Primer name & Sequence 5'-3' & Modifications & $\begin{array}{l}\text { Expected product } \\
\text { size }(\mathrm{pb})\end{array}$ & $\operatorname{Tm}\left({ }^{\circ} \mathrm{C}\right)$ \\
\hline \multicolumn{5}{|l|}{ T. circumcincta } \\
\hline TC SNP200 F & СACTCTTTCTGTACACCAATTG & {$[B \operatorname{tn}] 5$} & 128 & 60 \\
\hline Tc SNP200 R & AGTGATTGAGATCGCCATAA & - & & \\
\hline TC SNP167 F & CAAAATTCGCGAGGAGTAT & - & 276 & 60 \\
\hline Tc SNP167 R & TTCTACCAATTGGTGTACAGAAAG & {$[B \operatorname{tn}] 5$} & & \\
\hline \multicolumn{5}{|l|}{ T. colubriformis } \\
\hline Tri SNP200 F & TACTITATCAGTCCATGAGCTGG & {$[B \operatorname{tn}] 5$} & 128 & 62 \\
\hline Tri SNP200 R & ATGGTTGAGATCTCCATAGGTTG & - & & \\
\hline \multicolumn{5}{|l|}{ Sequencing } \\
\hline Tc $200 \mathrm{Seq}$ & AGAGCTTCATTATCGATG & - & - & 60 \\
\hline Tc $167 \mathrm{Seq}$ & CGGATAGAATCATGGCT & - & - & 60 \\
\hline Tri 200 Seq & AGAGCTTCGTTATCGATGCA & - & - & 62 \\
\hline
\end{tabular}


According to the results of FECRT, and taking into account the resistant and borderline flocks together, 40\% of flocks were resistant to one drug. Resistance to ABZ was observed in $12.5 \%(1 / 8)$ of the flocks and to IVM in $44.4 \%$ (4/9). One flock (10\%) was resistant to both drugs.

Before treatment, the most frequent species were $T$. circumcincta (43-55\% of larvae) and Trichostrongylus sp (38-47\% of larvae), however, other GIN species such as H. contortus, Bunostomun sp, Nematodirus sp, Chabertia ovina and Cooperia oncophora were also observed at a lower percentage (1-13\% of larvae) (Table 2). After treatment, only $T$. circumcincta and Trichostrongylus sp were identified, with the exception of farm 7 where Bunostomun sp was also identified (6\% of larvae) (Tables 3 and 4).

\section{$\beta$-Tubulin allele frequencies}

The assays were capable of detecting BZ resistanceassociated SNPs because, with gDNA from resistant adult samples, included as a positive control, the mean frequencies of the resistant allele at codon 200 were $89 \%$ in T. circumcincta and $92 \%$ in T. colubriformis.

\section{Allele frequencies before treatment}

In relation to $T$. circumcincta, the resistant allele was not found in any of the flocks tested at codons 167, 198 or 200. In T. colubriformis, the resistant allele was found in $11.1 \%(1 / 9)$ of the flocks at codon 198, with a very low percentage $(6.8 \%)$. At codon 200 , the resistant allele was found in $33.3 \%$ (3/9) of the flocks, with high frequencies ranging from 48.5 to $87.8 \%$ (Table 2).

\section{Allele frequencies after treatment with $A B Z$}

For $T$. circumcincta, after the administration of $A B Z$, the percentage of flocks with the resistant allele at codon 167 was $16.7 \%(1 / 6)$, with a frequency of $23.8 \%$. The resistant allele at codon 198 was found in one out of five flocks (20\%) with a frequency of $13.2 \%$. On the other hand, the resistant allele carrying SNP200 was not found in any flock.

In relation to $T$. colubriformis, the resistant allele at codon 198 was found in $25 \%$ (2/8) of flocks, with values of 5.5 and $19 \%$, and at position 200 in $75 \%(6 / 8)$ of flocks with very high frequencies, between 89.8 and 99.3\% (Table 3).

\section{Allele frequencies after treatment with IVM}

After treatment with IVM, for T. circumcincta, the resistant allele carrying SNP167 was found in $11.1 \%$ of the flocks $(1 / 9)$ but with a very low frequency $(6.1 \%)$. At codons 198 and 200, the resistant allele was not found in any sample.

For T. colubriformis, the SNP198 was found in 3 of the 8 flocks (37.5\%) at low frequency, between 6.1 and $14 \%$. At codon 200, all flocks in which the determination was done carried the resistant allele, with frequencies ranging from 23.3 to $100 \%$ (Table 4).

\section{Discussion}

This study describes the frequencies of the resistant alleles present in the gene encoding isotype- $1 \beta$-tubulin of field populations of GIN, collected before and after treatment with ABZ or IVM. The resistance status of these flocks situated in the Northwest of Spain was firstly determined in vivo by means of the FECRT.

Since the FECRT cannot detect low resistance levels, especially under field conditions when the infections by GIN are typically of mixed species composition [26], determining the frequency of resistant alleles in pools of L3 could be an alternative to or proxy for the FECRT, with the added benefit, if sufficiently robust and repeatable, of not needing to treat animals to determine resistance status. Since the resistant phenotype is only

Table 2 Allele frequencies before treatment for T. circumcincta SNPs 167 and 198/200, and T. colubriformis SNP 198/200, and morphological identification

\begin{tabular}{|c|c|c|c|c|c|c|c|c|c|c|c|c|}
\hline \multirow[t]{2}{*}{ Farm } & \multicolumn{2}{|c|}{ T. colubriformis } & \multicolumn{3}{|c|}{ T. circumcincta } & \multicolumn{7}{|c|}{$\%$ L3 species } \\
\hline & $\%$ SNP200 & \% SNP198 & \% SNP200 & \% SNP198 & \% SNP167 & $\overline{T C}$ & Tri & $\mathrm{HC}$ & $\mathrm{Bu}$ & $\mathrm{Ne}$ & $\mathrm{Ch}$ & $\mathrm{Co}$ \\
\hline 1 & 0 & 2.5 & 0 & 0 & 0 & 51 & 42 & 4 & 1 & 2 & 0 & 0 \\
\hline 2 & 51.6 & 6.8 & 0 & 0 & - & 49 & 47 & 3 & 0 & 1 & 0 & 0 \\
\hline 3 & - & - & 3.4 & 0 & 0 & 52 & 45 & 2 & 0 & 0 & 0 & 1 \\
\hline 4 & 0 & 3.3 & 0 & 0 & 2.6 & 43 & 41 & 2 & 12 & 1 & 2 & 0 \\
\hline 5 & 0 & 0 & 0 & 0 & 2.8 & 55 & 42 & 2 & 0 & 0 & 0 & 0 \\
\hline 6 & 3.1 & 0 & 0 & 0 & 2.8 & 48 & 38 & 0 & 1 & 0 & 13 & 0 \\
\hline 7 & 2 & 2 & 0 & 2 & 3.1 & 46 & 43 & 0 & 3 & 0 & 8 & 0 \\
\hline 8 & 87.8 & 3.9 & - & - & 0 & - & - & - & - & - & - & - \\
\hline 9 & 0 & 3.1 & 0 & 0 & - & 54 & 46 & 0 & 0 & 0 & 0 & 0 \\
\hline 10 & 48.5 & 0 & 0 & 0 & - & 53 & 46 & 0 & 1 & 0 & 0 & 0 \\
\hline
\end{tabular}

Tc: T. circumcincta, Tri: Trichostrongylus spp, Hc: H. contortus, Bu: Bunostomun spp, Ne: Nematodirus spp, Ch: Chabertia ovina and Co: Cooperia oncophora. -: Failed samples 
Table 3 Allele frequencies after ABZ treatment for T. circumcincta SNPs 167 and 198/200, and T. colubriformis SNP 198/200, and morphological identification

\begin{tabular}{|c|c|c|c|c|c|c|c|c|c|c|}
\hline \multirow[t]{2}{*}{ Farm } & \multirow{2}{*}{$\begin{array}{l}\% \text { Egg } \\
\text { reduction }\end{array}$} & \multirow{2}{*}{$\begin{array}{l}\text { R/S } \\
\text { classification }\end{array}$} & \multicolumn{2}{|c|}{ T. colubriformis } & \multicolumn{3}{|c|}{ T. circumcincta } & \multicolumn{3}{|c|}{ \% L3 species } \\
\hline & & & $\%$ SNP200 & \% SNP198 & $\%$ SNP200 & \% SNP198 & \% SNP167 & Tc & Tri & $\mathrm{Bu}$ \\
\hline 1 & 91.4 & Borderline & 1.7 & 4.8 & 2.9 & 0 & 3 & 50 & 50 & 0 \\
\hline 2 & 98.9 & $S$ & 97.6 & 3 & 0 & 0 & 23.8 & 55 & 45 & 0 \\
\hline 3 & 100 & $\mathrm{~S}$ & 96 & 1.5 & 0 & 13.2 & 4.4 & 56 & 44 & 0 \\
\hline 4 & 98.5 & $S$ & 99.3 & 5.5 & 0 & 0 & - & 58 & 42 & 0 \\
\hline 5 & 100 & S & 89.8 & 0 & 0 & 0 & 4.8 & 52 & 48 & 0 \\
\hline 6 & 99.3 & $\mathrm{~S}$ & 1.3 & 3 & - & - & - & 62 & 38 & 0 \\
\hline 7 & 100 & S & 90.6 & 19 & - & - & 0 & 47 & 47 & 6 \\
\hline 10 & 100 & $S$ & 97.2 & 4.8 & - & - & 0 & 38 & 62 & 0 \\
\hline
\end{tabular}

Tc: T. circumcincta, Tri: Trichostrongylus spp and Bu: Bunostomun spp. -: Failed samples

detected by the FECRT when the frequency of resistant alleles in the population is over 25\% [22], the FECRT only represents an estimate of the resistance in a flock naturally infected by GIN.

In this study, we used pyrosequencing to determine the resistant allele frequency in the gene encoding isotype-1 $\beta$-tubulin at codons 167, 198 and 200 of $T$. circumcincta and codons 198 and 200 of T. colubriformis, in DNA samples from pools of L3 collected before and after treatment. These species were the most frequent detected in all sampled flocks and represent the $84-100 \%$ of the GIN burden on all of them.

According to Kwa et al. [14] and Elard and Humbert [27], the mutation of Phe to Tyr at position 200 of $\beta$ tubulin isotype 1 is the most important mechanism responsible for conferring resistance to BZ. According to Silvestre and Cabaret [15], this same mutation at codon 167 is also involved in the development of resistance in the absence of the mutation at position 200. The SNP167 is rare in field populations, but for T. circumcincta may account for the survival of parasites which do not carry the mutation at codon 200 [15]. In the present study, this SNP was present before treatment; after treatment with ABZ, it was only shown in one susceptible flock with a low frequency of $23.8 \%$ and, therefore, we do not correlate it with AR. However, Demeler et al. [28] found resistant alleles at codons 167 and 200, but not at 198 in an Ostertagia ostertagi BZ resistant isolate, indicating that $\mathrm{BZ}$ treatment does not necessarily select for the SNP198. In the present study, the SNP at codon 198 was not found before treatment in T. circumcincta and, after administration of ABZ, only one susceptible flock, with a low frequency (13.2\%) was detected in the five flocks tested. Our results regarding the SNP198 in T. circumcincta are not conclusive, due to the low number of analyses. However, Ghisi et al. [16] found the SNP198 in 90\% of resistant isolates of $H$. contortus which did not carry the mutation in codon 200. Therefore, SNP198 has been related to $\mathrm{BZ}$ resistance. In $T$. colubriformis, before treatment, SNP198 was only found in one flock with a very low frequency $(6.8 \%)$, and after treatment with

Table 4 Allele frequencies after IVM treatment for T. circumcincta SNPs 167 and 198/200, and T. colubriformis SNP 198/200, and morphological identification

\begin{tabular}{|c|c|c|c|c|c|c|c|c|c|}
\hline \multirow[t]{2}{*}{ Farm } & \multirow{2}{*}{$\begin{array}{l}\% \text { Egg } \\
\text { reduction }\end{array}$} & \multirow{2}{*}{$\begin{array}{l}\text { R/S } \\
\text { classification }\end{array}$} & \multicolumn{2}{|c|}{ T. colubriformis } & \multicolumn{3}{|c|}{ T. circumcincta } & \multicolumn{2}{|c|}{ \% L3 species } \\
\hline & & & $\%$ SNP200 & $\%$ SNP198 & $\%$ SNP200 & \% SNP198 & $\%$ SNP167 & $\overline{T c}$ & Tri \\
\hline 1 & 37.3 & $\mathrm{R}$ & 23.3 & 6.4 & 0 & 0 & 0 & 63 & 37 \\
\hline 2 & 100 & S & 97.3 & 0 & 0 & 0 & 0 & 54 & 46 \\
\hline 3 & 86.0 & $\mathrm{R}$ & 79.2 & 14 & 0 & 0 & 2.4 & 41 & 59 \\
\hline 4 & -11.9 & $\mathrm{R}$ & 94.7 & 0 & 0 & 0 & 0 & 60 & 40 \\
\hline 5 & 99.3 & S & 93.7 & 1 & - & - & 0 & 50 & 50 \\
\hline 6 & 100 & S & - & - & 0 & 0 & 3 & 54 & 46 \\
\hline 7 & 95.9 & S & 100 & 14 & 0 & 0 & 0 & 70 & 30 \\
\hline 8 & 100 & S & 89.9 & 6.1 & - & - & 0 & - & - \\
\hline 9 & 93.5 & Borderline & 95 & 2.4 & 0 & 0 & 6.1 & 44 & 56 \\
\hline
\end{tabular}


ABZ in two different flocks with frequencies of 5.5 and $19 \%$.

Therefore, in the present study, the frequencies of resistant alleles carrying SNPs 167 and 198 in T. circumcincta, and SNP 198 in T. colubriformis, are either zero or very low and consequently we did not find any relation between them and AR status/phenotype.

In relation to the SNP200 in T. circumcincta, this was not found before or after treatment with ABZ. These results are in agreement with the previous study of Martinez-Valladares et al. [29], who did not describe any resistant allele at codons 167, 198 and 200 in individual T. circumcincta L3 collected from flocks in the same study area (León, Spain). Due to the absence of resistant alleles, but also because we only found one flock with borderline resistance to $\mathrm{ABZ}$, we cannot conclude that there is an association between resistance and resistant allele frequency at codon 200 in $T$. circumcincta. On the other hand, Skuce et al. [25], after studying different strains of $T$. circumcincta, reported a resistant allele frequency of $64.9 \%$ in a multidrug-resistant strain (MTci5) at codon 200 and $0 \%$ in a susceptible strain (MTci1).

However, the results shown in the current study for the frequencies at SNP 200 in T. colubriformis are totally different. Before treatment with $\mathrm{ABZ}$, the resistant allele was present in $33.3 \%$ of the farms tested, with values between 48.5 and $87.8 \%$ (Mean $=62.6 \%$ ) and, after ABZ treatment, in $75 \%$ of the farms, with very high allele frequencies, ranging from 89.8 to $99.3 \%$ (Mean =95.1\%). However, these data are not consistent with the results of the FECRT, since we found high levels of the resistant allele in susceptible farms classified according to the FECRT. This result could be due to the resistant allele(s) being diluted in the population because of the presence of other (susceptible) species, before treatment. The FECRT can only detect resistance when $\sim 25 \%$ of the total population is resistant. The resistant allele at codon 200 has been previously reported in different BZ resistant strains of $T$. colubriformis [30, 31] and also in Trichostrongylus axei adult worms, with a frequency of $63 \%$ recovered from lambs after treatment with BZ [32].

In the current study, we also determined the frequency of the resistant alleles for these species before and after the administration of IVM. Freeman et al. [33] were the first authors to describe a relation between resistance to IVM and the $\beta$-tubulin gene in $H$. contortus. These authors found a marked alteration in the amphid neurons, which are formed by bundles of microtubules, heterodimers of $\alpha$-tubulin and $\beta$-tubulin in IVM resistant strains of $H$. contortus. Moreover, Mottier and Prichard [17] reported that repetitive use of IVM and moxidectin in $H$. contortus strains produced changes in the frequency of the alleles at codons 167, 198 and 200 of $\beta$-tubulin.
After the administration of IVM, in T. circumcincta the resistant allele was not found at codons 198 and 200. At position 167, the resistant allele was found in only one flock but with a very low frequency of $6.1 \%$. These results are in agreement with a previous study in which none of these SNPs (167, 198 and 200) were detected in T. circumcincta when sheep were treated with IVM; the authors concluded that other molecular mechanisms than beta-tubulin could be implicated in the development of resistance against IVM in this case [29]. The present study would add further evidence to support this hypothesis, since 4 out of 9 studied flocks were resistant or borderline to IVM in the absence of the acknowledged resistant alleles. Therefore, we suggest that $T$. circumcincta is not responsible for the presence of resistance in these flocks and/or there could be different mechanisms or genes implicated in resistance development, especially to IVM. Previous studies suggest that the F200Y mutation could be related to IVM resistance in different species as observed by Njue et al. [34] in IVM-resistant strains of C. oncophora and by Eng and Prichard [35] in Onchocerca volvulus.

In contrast, for T. colubriformis, the frequency of the resistant allele at codon 200 after the administration of IVM was between 23.3 and $100 \%$ (Mean $=82.6 \%)$. These data suggest that there could be an association between the SNP 200 in T. colubriformis and IVM resistance. However, according to FECRT, only 4 flocks were resistant or borderline resistant to this drug. The effect of the resistant allele could be diluted by the presence of other susceptible species, like it happened after the treatment with ABZ. Recently, Ashraf et al. [36] reported that that IVM binds to $H$. contortus $\alpha$ and $\beta$ tubulins at low micromolar affinities and stabilizes the microtubules. However, in a different study, Ashraf et al. [37] concluded that the SNPs 167 and 200 cause no difference in the polymerization of wild and mutant tubulins, and therefore, neither of the SNPs reduced IVM binding. The hypothesis is that the SNPs 167 and 200 could be part of a signaling mechanism that results in over expression of P-glycoproteins, which ultimately leads to IVM resistance [37].

In relation to these results, we suggest that the presence of resistance is more common than the FECRT indicates. Therefore using only this in vivo test we could obtain false negatives. The percentage of resistant alleles show a more detailed perspective on the presence of resistance at the molecular level; in consequence the level of resistance of a strain will depend on the percentage of resistant alleles in each species. Therefore, it would be worthy monitoring the percentage of resistant alleles after the FECRT, in susceptible flocks to avoid the development of resistance to BZs, and in resistant ones to dilute the percentage of resistant alleles, in both cases applying different management practices at the same time. 


\section{Conclusions}

In conclusion, comparing the results of FECRT and pyrosequencing, we suggest that the presence of resistance is more common than expected and that would have been declared using FECRT alone. The SNP200 in $T$. circumcincta is not present in the Spanish nematode populations tested although its association with resistance has been described in others field isolates from other countries, most notably, UK [25] and France [31]. In contrast, in T. colubriformis, the SNP200 is present on multiple farms and at high frequency before (between 48.5 and $87.8 \%$ ) and after treatment with $\mathrm{ABZ}$ (mean in positive flocks $=95.1 \%)$ and IVM (mean $=82.6 \%)$. In relation to the other SNPs, 167 and 198, these were not detected in most of the analyses, so were either not present or present at very low frequency i.e. below the sensitivity of detection of the pyrosequencing assays used. Therefore, we cannot conclude that these SNPs are related with ABZ and/or IVM resistance in any of the Spanish GIN populations tested.

\section{Abbreviations}

ABZ: Albendazole; AR: Anthelmintic resistance; BZ: Benzimidazole BZs: Benzimidazoles; EPG: Eggs per gram of faeces; FECRT: Faecal egg count reduction test; GIN: Gastrointestinal nematodes; IVM: Ivermectin; L3: Third stage larvae; MLs: Macrocyclic lactones; SNP: Single nucleotide polymorphism; WAAVP: World association for the advancement of veterinary parasitology

\section{Acknowledgements}

This study has been funded by the national project INIA (Instituto Nacional de Investigaciones Agrarias: RTA2013-00064-C02-02) of the Ministry of Economy and Competitiveness (Ministerio de Economía y Competitividad), the European Regional Development Fund (Fondos Feder), the Spanish "Ramón y Cajal" Programme of the Ministry of Economy and Competitiveness (MMV RYC-2015-18368), and the Cooperativa Bajo Duero, COBADU. We acknowledge support of the publication fee by the CSIC Open Access Publication Support Initiative through its Unit of Information Resources for Research (URICI). The authors would like to thank all animal owners for their willingness to collaborate in the study.

\section{Funding}

Not applicable.

\section{Availability of data and materials}

The datasets supporting the conclusions of this article are presented in the main paper (Tables).

\begin{abstract}
Authors' contributions
MEB performed DNA extractions, PCR amplification, data analysis, and drafted the manuscript. FARV and CGL collected samples and revised the manuscript. PJS and LN performed the pyrosequencing and revised the manuscript. MMV designed and supervised the experiment, collected samples and drafted manuscript. All authors read and approved the final manuscript.
\end{abstract}

\section{Competing interests}

The authors declare that they have no competing interests.

\section{Consent for publication}

Not applicable.

Ethics approval and consent to participate Not applicable.

\section{Publisher's Note}

Springer Nature remains neutral with regard to jurisdictional claims in published maps and institutional affiliations.

\section{Author details}

${ }^{1}$ Departamento de Sanidad Animal, Facultad de Veterinaria, Universidad de León, Campus de Vegazana s/n, 24071 León, Spain. ${ }^{2}$ Instituto de Ganadería de Montaña (CSIC-Universidad de León), Finca Marzanas, Grulleros, 24346 León, Spain. ${ }^{3}$ Moredun Research Institute, Pentland Science Park, Bush Loan, Edinburgh, UK.

Received: 27 April 2016 Accepted: 16 March 2017

Published online: 20 March 2017

\section{References}

1. Kloosterman A, Parmentier HK, Ploeger HW. Breeding cattle and sheep for resistance to gastrointestinal nematodes. Parasitol Today. 1992;8:330-5.

2. Kaminsky R, Ducray P, Jung M, Clover R, Rufener L, Bouvier J, Weber SS, Wenger A, Wieland-Berghausen S, Goebel T, Gauvry N, Pautrat F, Skripsky T, Froelich O, Komoin-Oka C, Westlund B, Sluder A, Maser P. A new class of anthelmintics effective against drug-resistant nematodes. Nature. 2008;452:176-80.

3. Little PR, Hodges A, Watson TG, Seed JA, Maeder SJ. Field efficacy and safety of an oral formulation of the novel combination anthelmintic, derquantel-abamectin, in sheep in New Zealand. N Z Vet J. 2010;58:121-9.

4. Scott I, Pomroy WE, Kenyon PR, Smith G, Adlington B, Moss A. Lack of efficacy of monepantel against Teladorsagia circumcincta and Trichostrongylus colubriformis. Vet Parasitol. 2013;198:166-71.

5. Dobson RJ, Hosking BC, Jacobson CL, Cotter JL, Besier RB, Stein PA, Reid SA. Monepantel resistance reported on Dutch sheep farms. Vet Rec. 2014;175:418.

6. Mederos AE, Banchero GE, Ramos Z. First report of monepantel Haemonchus contortus resistance on sheep farms in Uruguay. Parasit Vectors. 2014;7:598.

7. Van den Brom R, Moll L, Kappert C, Vellema P. Haemonchus contortus resistance to monepantel in sheep. Vet Parasitol. 2015;209:278-80.

8. Kaplan RM, Klei TR, Lyons ET, Lester G, Courtney CH, French DD, Tolliver SC, Vidyashankar AN, Zhao Y. Prevalence of anthelmintic resistant cyathostomes on horse farms. J Am Vet Med Assoc. 2004:225:903-10.

9. Rojo-Vázquez FA, Hosking BC. A telephone survey of internal parasite control practices on sheep farms in Spain. Vet Parasitol. 2013;192:166-72.

10. Lubega GW, Prichard RK. Specific interaction of benzimidazole anthelmintics with tubulin: high-affinity binding and benzimidazole resistance in Haemonchus contortus. Mol Biochem Parasitol. 1990:38:221-32.

11. Lubega GW, Prichard RK. Beta-tubulin and benzimidazole resistance in the sheep nematode Haemonchus contortus. Mol Biochem Parasitol. 1991;47:129-37

12. Lubega GW, Prichard RK. Interaction of benzimidazole anthelmintics with Haemonchus contortus tubulin: binding affinity and anthelmintic efficacy. Exp Parasitol. 1991:73:203-13.

13. Coles GC, Klei TR. Animal parasites, politics and agricultural research. Parasitol Today. 1995;11:276-8

14. Kwa MS, Veenstra JG, Roos MH. Benzimidazole resistance in Haemonchus contortus is correlated with a conserved mutation at amino acid 200 in beta-tubulin isotype 1. Mol Biochem Parasitol. 1994;63:299-303.

15. Silvestre A, Cabaret J. Mutation in position 167 of isotype $1 \beta$-tubulin gene of Trichostrongylid nematodes: role in benzimidazole resistance? Mol Biochem Parasitol. 2002;120:297-300.

16. Ghisi M, Kaminsky R, Mäser P. Phenotyping and genotyping of Haemonchus contortus isolates reveals a new putative candidate mutation for benzimidazole resistance in nematodes. Vet Parasitol. 2007:144:313-20.

17. Mottier ML, Prichard RK. Genetic analysis of a relationship between macrocyclic lactone and benzimidazole anthelmintic selection on Haemonchus contortus. Pharmacogenet Genomics. 2008;18:129-40.

18. Sutherland IA, Leathwick DM. Anthelmintic resistance in nematode parasites of cattle: a global issue? Trends Parasitol. 2011:27:176-81.

19. Martínez-Valladares M, Geurden T, Bartram DJ, Martínez-Pérez JM, RoblesPérez D, Bohórquez A, Florez E, Meana A, Rojo-Vázquez FA. Resistance of gastrointestinal nematodes to the most commonly used anthelmintics in sheep, cattle and horses in Spain. Vet Parasitol. 2015:211:228-33.

20. Waller PJ. Anthelmintic in nematodes parasites of sheep. In: Russel GE, editor. Agricultural zoology reviews, Vol. 1. Poteland, UK: Intercept; 1986. p. 33-7. 
21. Jackson F, Coop RL. The development of anthelmintic resistance in sheep nematodes. Parasitology. 2010;120(Suppl):95-107.

22. Martin PJ, Anderson N, Jarrett RG. Detecting benzimidazole resistance with faecal egg count reduction tests and in vitro assays. Aust Vet J. 1989;66:236-40.

23. MAFF (Ministry of Agriculture, Fisheries and Food). Manual of veterinary Parasitology laboratory techniques. 3rd ed. London: GB; 1986.

24. Coles GC, Bauer C, Borgsteede FH, Geerts S, Klei TR, Taylor MA, Waller PJ. World Association for the Advancement of Veterinary Parasitology (W.A.A.V.P.) methods for the detection of anthelmintic resistance in nematodes of veterinary importance. Vet Parasitol. 1992:44:35-44.

25. Skuce P, Stenhouse L, Jackson F, Hypša V, Gilleard J. Benzimidazole resistance allele haplotype diversity in United Kingdom isolates of Teladorsagia circumcincta supports a hypothesis of multiple origins of resistance by recurrent mutation. Int J Parasitol. 2010;40:1247-55.

26. McKenna PB. Further potential limitations of the undifferentiated faecal egg count reduction test for the detection of anthelmintic resistance in sheep. N Z Vet J. 1997:45:244-6.

27. Elard L, Humbert JF. Importance of the mutation of amino acid 200 of the isotype 1 beta-tubulin gene in the benzimidazole resistance of the smallruminant parasite Teladorsagia circumcincta. Parasitol Res. 1999;85:452-6.

28. Demeler J, Krüger N, Krücken J, von der Heyden VC, Ramünke S, Küttler U, Miltsch S, López Cepeda M, Knox M, Vercruysse J, Geldhof P, Harder A, von Samson-Himmelstjerna G. Phylogenetic characterization of $\beta$-tubulins and development of pyrosequencing assays for benzimidazole resistance in cattle nematodes. PLoS One. 2013;8:e70212.

29. Martínez-Valladares M, Donnan A, Geldhof P, Jackson F, Rojo-Vázquez FA, Skuce $P$. Pyrosequencing analysis of the beta-tubulin gene in Spanish Teladorsagia circumcincta field isolates. Vet Parasitol. 2012;184:371-6.

30. Grant WN, Mascord LJ. Beta-tubulin gene polymorphism and benzimidazole resistance in Trichostrongylus colubriformis. Int J Parasitol. 1996;26:71-7.

31. Silvestre A, Humbert JF. Diversity of benzimidazole-resistance alleles in populations of small ruminant parasites. Int J Parasitol. 2002;32:921-8.

32. Palcy C, Silvestre A, Sauve C, Cortet J, Cabaret J. Benzimidazole resistance in Trichostrongylus axei in sheep: long-term monitoring of affected sheep and genotypic evaluation of the parasite. Vet J Jan. 2010;183:68-74.

33. Freeman AS, Nghiem C, Li J, Ashton FT, Guerrero J, Shoop WL, Schad GA. Amphidial structure of ivermectin-resistant and susceptible laboratory and field strains of Haemonchus contortus. Vet Parasitol. 2003;110:217-26.

34. Njue Al, Prichard RK. Cloning two full-length beta-tubulin isotype cDNAs from Cooperia oncophora, and screening for benzimidazole resistanceassociated mutations in two isolates. Parasitology. 2003;127:579-88.

35. Eng JK, Prichard RK. A comparison of genetic polymorphism in populations of Onchocerca volvulus from untreated-and ivermectin-treated patients. Mol Biochem Parasitol. 2005;142:193-202.

36. Ashraf S, Beech RN, Hancock MA, Prichard RK. Ivermectin binds to Haemonchus contortus tubulins and promotes stability of microtubules. Int J Parasitol. 2015:45:647-54.

37. Ashraf S, Mani T, Beech RN, Prichard RK. Macrocyclic lactones and their relationship to the SNPs related to benzimidazole resistance. Mol Biochem Parasitol. 2015;201:128-34.

\section{Submit your next manuscript to BioMed Central and we will help you at every step:}

- We accept pre-submission inquiries

- Our selector tool helps you to find the most relevant journal

- We provide round the clock customer support

- Convenient online submission

- Thorough peer review

- Inclusion in PubMed and all major indexing services

- Maximum visibility for your research

Submit your manuscript at www.biomedcentral.com/submit 